\title{
The alcohol used for cleansing the venipuncture site does not jeopardize blood and plasma alcohol measurement with head-space gas chromatography and an enzymatic assay
}

\author{
Giuseppe Lippi*1,2, Ana-Maria Simundic ${ }^{1,3}$, Giacomo Musile4, Elisa Danese², Gianluca Salvagno², Franco Tagliaro 4 \\ ${ }^{1}$ Working Group for Preanalytical Phase (WG-PRE), European Federation for Clinical Chemistry and Laboratory Medicine (EFLM) \\ ${ }^{2}$ Section of Clinical Biochemistry, University of Verona, Verona, Italy \\ ${ }^{3}$ Department of Medical Laboratory Diagnostics, University Hospital Sveti Duh, Zagreb, Croatia \\ ${ }^{4}$ Department of Diagnostics and Public Health, Unit of Forensic Medicine, University of Verona, Verona, Italy \\ *Corresponding author: giuseppe.lippi@univr.it
}

\begin{abstract}
Introduction: This study aimed to establish whether an alcoholic antiseptic, wiped or not before venipuncture, may jeopardize alcohol testing with a commercial enzymatic assay and a reference head-space gas chromatography (GC) technique.

Materials and methods: Venous blood was collected from 23 healthy volunteers, with two sequential procedures. In the first blood collection, $2 \mathrm{~mL}$ of alcoholic antiseptic ( $0.5 \%$ chlorhexidine, $70 \%$ ethanol) were place on a gauge pad, the venipuncture site of right arm was cleaned but the antiseptic was not let to dry before phlebotomy. In the second blood collection, $2 \mathrm{~mL}$ of the same alcoholic antiseptic were placed on another gauge pad, the venipuncture site of left harm was cleaned and the antiseptic was accurately cleansed before phlebotomy. Ethanol was measured with a reference $\mathrm{GC}$ technique in whole blood and EDTA plasma, and a commercial enzymatic assay in EDTA plasma.

Results: No subject complained about feeling a particular itchy sensation when the alcohol was not wiped before puncturing the vein. The concentration of alcohol in all EDTA plasma samples was always lower than the limit of detection of the enzymatic assay (i.e., $2.2 \mathrm{mmol} / \mathrm{L} ; 0.1 \mathrm{~g} / \mathrm{L})$. Similarly, alcohol concentration was also undetectable using a reference $\mathrm{GC}$ technique (i.e., $<0.22 \mathrm{mmol} / \mathrm{L} ; 0.01 \mathrm{~g} / \mathrm{L}$ ) in EDTA plasma and whole blood.

Conclusion: It seems reasonable to conclude that using ethanol-containing antiseptics before venipuncture may not be causes of spurious or false positive results of alcohol measurement at least when ideal venipunctures can be performed.
\end{abstract}

Key words: laboratory testing; diagnostics; alcohol; errors; pre-analytical phase

\section{Introduction}

The collection of venous or arterial blood specimens is one of the most frequent practices in healthcare $(1,2)$, along with collection of clinical history, medical examination, blood pressure, heart rate and temperature measurement (3). Blood drawing is an essential practice for obtaining a suitable material (i.e., whole blood, serum or plasma), in which the vast majority of laboratory tests can be performed (4). Regardless of which of the many existing blood collection guidelines is followed by the phlebotomist, the need of accurate skin disinfection at venipuncture site is always considered a necessary activity for preventing bacteremia. More specifically, the guidelines of the World Health Organization (WHO) mandates that the venipuncture site should be cleaned with a $70 \%$ alcohol swab, and alcohol should then be allowed to dry for not less than 30 seconds before puncturing the vein (5). Alcohol is preferred to povidone iodine due to the fact that blood contamination with this latter antiseptic may generate falsely increased values of potassium, phosphorus or uric acid (5). The Clinical and Laboratory Standards Institute (CLSI) document H3-A6 also man- 
dates that skin disinfection with $70 \%$ isopropyl alcohol or ethanol is always necessary before venipuncture (6). As in the WHO guidelines, the CLSI also recommends that alcohol should be allowed to dry completely before inserting the needle into the vein (6). The Croatian Society of Medical Biochemistry and Laboratory Medicine has published national guidelines, which recommend that the venipuncture site should be accurately disinfected with $70 \%$ isopropyl alcohol or ethanol placed on sterile cotton or gauze pad before drawing blood (7). Notably, when blood alcohol measurement is ordered, the Croatian guidelines also recommend that non-alcoholic disinfectants should be used (i.e., ether or benzene). Finally, the guidelines of the Italian Society of Clinical Biochemistry and Clinical Molecular Biology (SIBioC) recommend that the skin at the venipuncture site should be cleansed with an appropriate alcoholic antiseptic, which should then be allowed to dry before puncturing the vein (8).

Besides its use in forensic medicine to provide corroborative evidence of impairment at the wheel, the laboratory measurement of alcohol in whole blood, serum or plasma is crucial for diagnosing alcohol abuse or toxicity $(9,10)$. Despite head-space gas chromatography (GC) remains the gold standard for measuring alcohol for forensic medicine purposes, alcohol assessment in routine clinical laboratories is usually accomplished by enzymatic techniques, most of which based on the alcohol dehydrogenase method $(11,12)$. The use of enzymatic assays in clinical practice is justified by many practical reasons, such as suitability for automation, shorter turnaround time (TAT), lower costs and no need for specialized personnel for running the test compared to GC.

Except for the recommendations of the Croatian Society of Medical Biochemistry and Laboratory Medicine, the indication of using ethanol or other alcoholic antiseptics before drawing blood is one common aspect of all other guidelines. Although two separate studies clearly demonstrated that the practice of avoiding to wipe alcohol is not associated with spurious hemolysis or sample dilution, the issue of potential sample contamination with the alcohol used for cleansing the venipunc- ture site remains a matter of debate $(13,14)$. The use of alcohol for cleaning the venipuncture site has been recognized as a possible source of contamination of blood specimens since 1976 (15). Moreover, it has also been demonstrated that alcohol could be absorbed through the intact skin of adult humans, thus leading to barely measurable blood alcohol levels, comprised between 0.01 - $0.04 \mathrm{mmol} / \mathrm{L}$ (16). The risk of contaminating blood specimens may be magnified when the phlebotomist does not allow the alcohol to dry for at least 30 seconds before puncturing the vein, a practice that is justified for preventing a prolonged placing of tourniquet and the ensuing risk of hemoconcentration and spurious increase of some measurable analytes in blood (17). Notably, this aspect may have substantial forensic implications, since the blood alcohol content (BAC) drink driving limit across Europe is on average $10.8 \mathrm{mmol} / \mathrm{L}$ (i.e., 0.5 $\mathrm{g} / \mathrm{L}$ ), but varies between $0 \mathrm{mmol} / \mathrm{L}$ in Romania, Slovakia, Hungary and Czech Republic, up to 17.4 $\mathrm{mmol} / \mathrm{L}$ (i.e., $0.8 \mathrm{~g} / \mathrm{L}$ ) in Malta and in the United Kingdom (18). Even more importantly, the BAC drink driving limit for novice drivers has been set to $0 \mathrm{mmol} / \mathrm{L}$ in many European countries including Croatia, Czech Republic, Germany, Hungary, Italy, Lithuania, Romania, Slovakia and Slovenia (18). Although some local forensic regulations currently discourage the use of alcoholic antiseptics for cleansing the skin before collecting blood for alcohol testing (19), the real world practice is often different from theory, with studies reporting that the use of alcohol-based antiseptics is actually commonplace when drawing blood for BAC (20). This is not surprising since the acquisition of blood tubes, integrated blood collection systems, as well as other phlebotomy tools such as (alcoholic) antiseptics, tourniquets, cotton or gauge pads, is now regulated by regional or national tenders in many countries worldwide, so that the local purchase of these materials for particular types of blood collections is no longer allowed by some hospital administrations (21).

Therefore, the aim of this study was to investigate whether the use of an alcoholic antiseptic, as well as the avoidance of wiping the alcohol before venipuncture, may both have an impact on plasma 
and whole blood alcohol measurement using a reference head-space GC technique and a routine enzymatic assay.

\section{Materials and methods}

\section{Study design and blood collection}

The study population consisted of 23 ostensibly healthy laboratory professionals ( 18 women and 5 men; mean age, $49 \pm 8$ years; body mass index, $24.7 \pm 4.7 \mathrm{~kg} / \mathrm{m}^{2}$ ), who voluntarily participated to this study. Two sequential procedures were followed for collecting venous blood from each volunteer, who had all abstained from ingesting food or drinking alcoholic beverages for at least 8 hours before the study. A first blood drawn was performed on the right arm, as follows. A pipette (Eppendorf Reference 100 - $1000 \mu \mathrm{L}$ pipette; Eppendorf, Marburg, Germany) was used to place $2 \mathrm{~mL}$ of alcoholic antiseptic $(0.5 \%$ chlorhexidine digluconate and $70 \%$ ethanol; Neoxinal Alcolico; Nuova Farmec, Verona, Italy) on a $30 \mathrm{~mm}$ diameter prepackaged gauge pad (Luigi Salivari SPA, Florence, Italy). This amount of alcohol corresponds to the approximate volume manually placed on the gauge pad by the nurses in our hospital. A tourniquet was applied, the venipuncture site was cleaned, the antiseptic was not let to dry and the needle was inserted into the vein within 5 seconds after cleansing. Blood was collected into a $6 \mathrm{~mL}$ evacuated blood tube containing $\mathrm{K}_{2}$ EDTA (Vacutest Kima, containing 10.8 mg spray $\mathrm{K}_{2}$ EDTA, Ref. 135400; Kima, Padova, Italy). A second blood drawn was then performed on the left arm, as follows. The same pipette (Eppendorf Reference) was used to place $2 \mathrm{~mL}$ of the same alcoholic antiseptic (Neoxinal Alcolico; Nuova Farmec, Verona, Italy) on another $30 \mathrm{~mm}$ diameter pre-packaged gauge pad (Luigi Salivari SPA, Florence, Italy). A tourniquet was applied, the venipuncture site was cleansed, but the antiseptic was accurately dried by using another $30 \mathrm{~mm}$ diameter cotton ball, using circular motions from centre to periphery, as recommended by the Croatian Society of Medical Biochemistry and Laboratory Medicine (7). The needle was finally inserted into the vein within 5 seconds after drying the antiseptic. Blood was collected into another $6 \mathrm{~mL}$ evacuated blood tube containing $\mathrm{K}_{2}$ EDTA (Kima, Padova, Italy).

For each venipuncture blood was collected directly into the evacuated blood tube through a 19 gauge straight needle. All procedures of the venipuncture (i.e., skin cleansing and obtaining blood) were standardized according to national guidelines $(8,22)$ and performed by the same expert phlebotomist. In all circumstances the needle was withdrawn from the vein after the blood tube had been completely filled and had been removed from the holder, as in an optimal venipuncture. Contact between needle and gauge pad was also carefully avoided throughout the venipuncture. Immediately after collection the blood tubes were mixed by 4-time gentle inversion. The blood in each primary tube was then divided in two identical aliquots, the first to be used for performing GC analysis, whereas the second was used for enzymatic testing. Both aliquots were always maintained capped until measurements. Alcohol testing was performed by the reference GC technique using both EDTA blood and EDTA plasma, whereas the enzymatic assessment was performed using EDTA plasma. The EDTA plasma was obtained after centrifugation of whole blood aliquots at $3000 \mathrm{~g}$ for $15 \mathrm{~min}$ at room temperature.

\section{Methods}

Ethanol concentration was measured with the reference CG technique, on both blood and plasma EDTA. Briefly, ethanol was first measured in whole blood, by opening the tube, and removing $100 \mu \mathrm{L}$ of whole blood. The specimen was then immediately recapped to prevent alcohol evaporation, was centrifuged to separate plasma from blood cells and ethanol concentration was finally measured on plasma EDTA. Alcohol measurement with the reference head-space GC technique was carried out using a Young Lin 6100 fully optimized head-space GC analyzer with advanced pneumatic control and flame ionization detector (Young Lin Instrument Co, Anyang, Korea). Both the EDTA plasma and whole blood were mixed 1:5 with an internal standard (tert-butyl alcohol at $0.0975 \mathrm{~g} / \mathrm{L}$; Carlo Erba Reagents, Cornaredo, Italy). A total vol- 
ume of $1250 \mu \mathrm{L}$ of sample was injected in the system. The temperature of the syringe was $80^{\circ} \mathrm{C}$ and the isotherm separation was carried out at $40{ }^{\circ} \mathrm{C}$. An internal quality standard (Carlo Erba Reagents, Cornaredo, Italy) with an ethanol concentration of $5.4 \mathrm{mmol} / \mathrm{L}$ was also measured every 5 test samples. The lower limit of detection of this technique is $0.22 \mathrm{mmol} / \mathrm{L}$ (i.e., $0.01 \mathrm{~g} / \mathrm{L}$ ).

The concentration of alcohol in plasma EDTA was also measured using a Roche Cobas 6000 analyzer (Roche Diagnostics GmbH, Mannheim, Germany), with an original Roche commercial reagent (Roche Diagnostics GmbH, Mannheim, Germany), based on the alcohol dehydrogenase method. In a previous study, the imprecision of this assay was found to be $2.4 \%$ at a plasma ethanol concentration of $31.6 \mathrm{mmol} / \mathrm{L}$ (23), whereas the measuring range of the assay has been declared to range between 2.2 - $108 \mathrm{mmol} / \mathrm{L}$ by the manufacturer.

All subjects signed a written consent for being recruited to this study, which was performed in accordance with the Declaration of Helsinki, under the terms of relevant local legislation, and was approved by the local Ethical Committee (University Hospital of Verona, Verona, Italy - SOPAV2, protocol number 35747; date of approval: 25 July 2016).

\section{Results}

No subject complained about feeling a particular itchy sensation when the alcohol was not wiped before puncturing the vein. The concentration of alcohol in all EDTA plasma samples was found to be always lower than the limit of detection of the enzymatic assay (i.e., $<2.2 \mathrm{mmol} / \mathrm{L} ;<0.10 \mathrm{~g} / \mathrm{L}$ ). Similarly, the alcohol concentration was also undetectable by the reference GC technique (i.e., $<0.22$ $\mathrm{mmol} / \mathrm{L} ;<0.01 \mathrm{~g} / \mathrm{L}$ ) in all EDTA plasma and whole blood specimens.

\section{Discussion}

Controversial evidence on alcohol measurement has been previously published when the venipuncture site is cleaned with alcohol-containing antiseptics.
The first alert about the possible interference from using alcohol antiseptics for cleansing the venipuncture site was published by Müller and Hundt in 1976 (15). In their elegant study, the authors collected three sequential evacuated blood tubes from each of 10 healthy volunteers, for measuring ethanol concentrations with GC. The skin was cleansed with an antiseptic containing 10\% chlorhexidine and $70 \%$ ethanol. Before alcohol evaporation had occurred, the needle was inserted into the vein and the first two blood tubes were completely filled, whereas the needle was withdrawn from the vein while the third blood tube was still aspirating. Importantly, the presence of ethanol on the skin did not produce a measurable concentration of blood alcohol in the first two tubes, whereas a measurable concentration of blood alcohol was found in the third tube in eight out of the ten subjects, with blood alcohol values between 0.4 - $743.9 \mathrm{mmol} / \mathrm{L}$.

In a following study, Goldfinger and Schaber measured alcohol concentration with an enzymatic assay in 25 emergency patients, after collecting blood with alcohol prep pad on one arm and non-alcoholcontaining germicidal solution on the other (24). No significant difference was appreciated in BAC obtained by either method of skin preparation.

Opposite results were published by Peek et al. (25). Briefly, the authors collected blood from 10 healthy volunteers during heavy drinking by either cleansing the venipuncture site with absolute ethanol on one arm or leaving the venipuncture site unswabbed on the opposite arm. Blood ethanol concentration was found to be significantly increased (up to $3.9 \mathrm{mmol} / \mathrm{L}$ ) in samples collected from the ethanol-cleansed arm compared to the unswabbed arm. Unlike these findings, the concentration of blood ethanol was unaltered when ethanol was replaced with isopropanol.

Mclvor and Cosbey measured BAC by means of head-space GC in 20 subjects, whose blood had been drawn after soaking the venipuncture site with ethanol, isopropyl alcohol or non-alcoholic cetrimide/chlorhexidine swab, and concluded that a modest ethanol interference could be appreciated by using alcohol-based skin cleansing swabs (26). Malingré studied the possible impact of 
cleansing the venipuncture site with an alcohol swab, by measuring blood ethanol concentration using both GC and an enzymatic assay in 50 patients aged $\leq 12$ years and in 20 healthy volunteers (27). In no sample the concentration of blood ethanol was found to be measurable (i.e., $<4.3 \mathrm{mmol} / \mathrm{L}$ in all cases). Controversial evidence was provided by Higuchi et al., who collected blood from 40 healthy subjects after cleansing the venipuncture site with either ethanol or saline (20). Although no blood uptake of ethanol or physiological saline could be recorded by the reference GC technique when both fluids were allowed to dry for $1 \mathrm{~min}$, a minimal alcohol intake (i.e., around $0.2 \mathrm{mmol} / \mathrm{L}$ ) was however noticed in $40 \%$ percent of subjects when the venipuncture was performed immediately after cleaning the skin with ethanol (i.e., within 5 seconds, as in our study). The rate of contamination considerably increased to $70 \%$ when the needle was voluntarily touched or swabbed by an ethanol-soaked cotton pad.

In an ensuing study, including five volunteers, Miller et al., showed that the use of skin prep pads containing 70\% isopropyl alcohol was not likely to generate false-positive blood ethanol levels using an enzymatic assay, since all the test results were found to be below the limit of detection of the assay (i.e., $1.1 \mathrm{mmol} / \mathrm{L}$ ) (28). Tucker and Trethewy carried out an interesting study, based on swabbing one arm with $70 \%$ isopropyl alcohol solution, allowed to completely dry before venipuncture, and the opposite arm with saline (29). Blood ethanol concentration measured with an enzymatic assay was not found to be statistically different between the two cleansing procedures. Opposite results were reported by another investigation carried out by Yigit and Arslan, who described the case of a 20-year-old male patient who crushed is head under a tree trunk and was brought to the hospital for urgent care (30). Blood testing upon patient admission to the emergency department showed a very high BAC (i.e., $98.3 \mathrm{mmol} / \mathrm{L}$ ), despite the patient denied having ingested any type of alcoholic beverage. Since the nurse who drew the blood admitted to have used alcoholic antiseptic for cleansing the venipuncture site, a second blood sample was collected after povidone-iodine swabbing of the skin. The $\mathrm{BAC}$ in this second sample was $0.07 \mathrm{mmol} / \mathrm{L}$.
Our results suggest that not letting an ethanolcontaining antiseptic to evaporate before venipuncture is not associated with measurable amount of alcohol in EDTA plasma or whole blood using both a conventional routine enzymatic assay with a limit of detection of $2.2 \mathrm{mmol} / \mathrm{L}$ (i.e., $0.1 \mathrm{~g} / \mathrm{L}$ ) and a reference $\mathrm{GC}$ technique (limit of detection of $0.22 \mathrm{mmol} / \mathrm{L} ; 0.01 \mathrm{~g} / \mathrm{L}$ ). These results obviously refer to conditions of an ideal venipuncture, performed by a skilled phlebotomist and following the local guidelines. Therefore, the practices of using alcoholic antiseptics, as well as that of not letting alcohol to evaporate before puncturing the vein, may be safe and relatively painless when alcohol testing is ordered for both clinical and forensic purposes and venipuncture guidelines are strictly followed.

According to our data, it seems hence reasonable to conclude that using ethanol-containing antiseptics before venipuncture may not be considered important cause of spurious or false positive results of alcohol measurement. Nevertheless, since it is likely a less than ideal venipuncture may still lead to contamination of collected blood with ethanol-containing antiseptics, as shown in some previous studies $(15,20)$, we suggest that the collection of blood samples for alcohol testing should be preferably repeated whenever occurring in one or more of the conditions listed in Table 1, and that it may be probably safer to let the alcohol evaporating before performing a venipuncture for forensic ethanol testing.

\section{Potential conflict of interest}

None declared.

TABLE 1. List of conditions potentially leading to contamination of blood tubes by alcohol-containing antiseptics

Conditions potentially leading to contamination of blood tubes by alcohol-containing antiseptics

Needle touched by cotton or gauge pad soaked with alcohol Needle swabbed by cotton or gauge pad soaked with alcohol Venipuncture performed with needle under pressure by cotton or gauge pad soaked with alcohol

Needle withdrawn from the vein while the blood tube was still aspirating 


\section{References}

1. Lavery I, Ingram P. Venepuncture: best practice. Nurs Stand 2005;19:55-65. https://doi.org/10.7748/ns2005.08.19.49.55. c3936

2. lalongo C, Bernardini S. Phlebotomy, a bridge between laboratory and patient. Biochem Med (Zagreb) 2016;26:1733. $h$ ttps://doi.org/10.11613/BM.2016.002

3. Booth KA. Clinical Procedures for Medical Assisting. McGrawHill, New York, USA; 2010. ISBN-13: 9780077340087.

4. Lippi G, Salvagno GL, Montagnana M, Franchini M, Guidi GC. Phlebotomy issues and quality improvement in results of laboratory testing. Clin Lab 2006;52:217-30.

5. World Health Organization. WHO guidelines on drawing blood: best practices in phlebotomy. WHO Press, Geneva, Switzerland; 2010.

6. Clinical and Laboratory Standards Institute. Procedures for collection of diagnostic blood specimens by venipuncture; approved guideline - 6th ed. CLSI document H3-A6. Wayne, PA: Clinical and Laboratory Standards Institute; 2007.

7. Nikolac N, Šupak-Smolčić V, Šimundić AM, Celap l; Croatian Society of Medical Biochemistry and Laboratory Medicine. Croatian Society of Medical Biochemistry and Laboratory Medicine: national recommendations for venous blood sampling. Biochem Med (Zagreb) 2013;23:242-54. https:// doi.org/10.11613/BM.2013.031

8. Lippi G, Caputo M, Banfi G, Buttarello M, Cerotti F, Daves M, et al. Recommendations for collection of venous blood. Biochim Clin 2008;32:569-77.

9. Lippi G, Plebani M. Pharmacogenomics of alcohol metabolism: implications for legal testing. Clin Chem Lab Med 2011;49:9-11. https://doi.org/10.1515/CCLM.2011.025

10. Church AS, Witting MD. Laboratory testing in ethanol, methanol, ethylene glycol, and isopropanol toxicities. J Emerg Med 1997;15:687-92. https://doi.org/10.1016/S07364679(97)00150-9

11. Tagliaro F, Lubli G, Ghielmi S, Franchi D, Marigo M. Chromatographic methods for blood alcohol determination. J Chromatogr 1992;580:161-90. https://doi.org/10.1016/03784347(92)80534-W

12. Logan BK, Lowrie KJ, Turri JL, Yeakel JK, Limoges JF, Miles $A K$, et al. Recommendations for toxicological investigation of drug-impaired driving and motor vehicle fatalities. J Anal Toxicol 2013;37:552-8. https://doi.org/10.1093/jat/bkt059

13. Salvagno GL, Danese E, Lima-Oliveira G, Guidi GC, Lippi G. Avoidance to wipe alcohol before venipuncture is not a source of spurious hemolysis. Biochem Med (Zagreb) 2013;23:201-5. https://doi.org/10.11613/BM.2013.023

14. Sarmah D, Sharma B, Sharma D, Mathew S. Alcohol Used as Disinfectant before Venipuncture does not Lead to Sample Haemolysis or Sample Dilution. J Clin Diagn Res 2016;10:BC16-8. https://doi.org/10.7860/ jcdr/2016/15967.7245

15. Müller FO, Hundt HKL. Letter: Ethyl alcohol: contamination of blood specimens. S Afr Med J 1976;50:91.

16. Turner P, Saeed B, Kelsey MC. Dermal absorption of isopropyl alcohol from a commercial hand rub: implications for its use in hand decontamination. J Hosp Infect 2004;56:287-90. https://doi.org/10.1016/j.jhin.2004.01.005
17. Lima-Oliveira G, Lippi G, Salvagno GL, Montagnana $M$, Picheth $G$, Guidi GC. The effective reduction of tourniquet application time after minor modification of the CLSI H03-A6 blood collection procedure. Biochem Med (Zagreb) 2013;23:308-15. https://doi.org/10.11613/BM.2013.037

18. European Transport Safety Council. Blood Alcohol Content (BAC) Drink Driving Limits across Europe. Available at: http://etsc.eu/blood-alcohol-content-bac-drink-driving-limits-across-europe. Last accessed, 1 April 2017.

19. Taylor LE, Oberman S, Oberman T, eds. Drunk Driving Defense, 7th Edition. Philadelphia: Aspen Publishers Inc; 2010.

20. Higuchi A, Kurihara R, Yoshimoto T, Sano T, Katsumata K, Ishii A, Katsumata Y. Problems in blood alcohol testing of severely injured drivers brought to emergency departments in Japan. Leg Med (Tokyo) 2005;7:299-305. https://doi. org/10.1016/j.legalmed.2005.05.003

21. Lippi G, Cornes MP, Grankvist K, Nybo M, Simundic AM; Working Group for Preanalytical Phase (WG-PRE); European Federation of Clinical Chemistry and Laboratory Medicine (EFLM). EFLM WG-Preanalytical phase opinion paper: local validation of blood collection tubes in clinical laboratories. Clin Chem Lab Med 2016;54:755-60. https://doi. org/10.1515/cclm-2015-1274

22. Lippi G, Mattiuzzi C, Banfi G, Buttarello M, Caputo M, Daves $M$, et al. Proposal of a checklist for venous blood collection. Biochim Clin 2013;37312-7.

23. Kocak FE, Isiklar OO, Kocak H, Meral A. Comparison of blood ethanol stabilities in different storage periods. Biochem Med (Zagreb) 2015;25:57-63. https://doi.org/10.11613/ BM.2015.006

24. Goldfinger TM, Schaber D. A comparison of blood alcohol concentration using non-alcohol- and alcohol-containing skin antiseptics. Ann Emerg Med 1982;11:665-7. https://doi. org/10.1016/S0196-0644(82)80260-6

25. Peek GJ, Marsh A, Keating J, Ward RJ, Peters TJ. The effects of swabbing the skin on apparent blood ethanol concentration. Alcohol Alcohol 1990;25:639-40. https://doi. org/10.1093/oxfordjournals.alcalc.a045060

26. Mclvor RA, Cosbey SH. Effect of using alcoholic and non-alcoholic skin cleansing swabs when sampling blood for alcohol estimation using gas chromatography. Br J Clin Pract 1990;44:235-6.

27. Malingré M, Ververs T, Bos S, van Kesteren C, van Rijn H. Alcohol swabs and venipuncture in a routine hospital setting: no effect on blood ethanol measurement. Ther Drug Monit 2005;27:4034. https://doi.org/10.1097/01.ftd.0000158868.74618.dd

28. Miller MA, Rosin A, Levsky ME, Gregory TJ, Crystal CS. Isopropyl alcohol pad use for blood ethanol sampling does not cause false-positive results. J Emerg Med 2007;33:9-10. https://doi.org/10.1016/j.jemermed.2006.10.005

29. Tucker A, Trethewy C. Lack of effect on blood alcohol level of swabbing venepuncture sites with $70 \%$ isopropyl alcohol. Emerg Med Australas 2010;22:9-12. https://doi.org/10.1111/ j.1742-6723.2009.01248.x

30. Yigit $O$, Arslan S. Swabbing skin with alcohol could change the blood alcohol level significantly. J Emerg Med 2012;43:718-9. https://doi.org/10.1016/j.jemermed.2011.07.025 